\title{
Functional Movements in Japanese Mini-Basketball Players
}

\author{
by \\ Kenji Kuzuhara ${ }^{1,4}$, Masashi Shibata ${ }^{2}$ Junta Iguchi ${ }^{3}$, Ryo Uchida
}

Functional movement screen (FMS) has been used to establish normative data and determine potential injury risk for young adults and athletes, but there are few data in elementary school-age children. The purpose of this study was to establish fundamental values for the FMS in elementary school-age mini-basketball players. Secondary purposes were to examine relationships between functional movement patterns and age, peak height velocity (PHV), and body mass index (BMI), and to compare functional movement patterns between boys and girls and between individuals with and without a history of injury. The mean composite FMS score was $16.5 \pm 2.2$ (16.5 \pm 2.4 for boys, $16.5 \pm 1.7$ for girls). The composite FMS score was positively correlated with age $(r=.312)$ and negatively correlated with the BMI $(r$ $=-.371)$. However, the FMS score was not correlated with PHV or with PHV age. The FMS score was not different between boys and girls or between individuals who reported a previous injury and those who did not. However, boys in the mini-basketball teams performed better than girls on the trunk stability push-up and rotary stability tests. Age and the body mass index were significantly associated with better and poorer functional movement, respectively.

Key words: functional movement screen, elementary school-aged children, injury risk, basketball players, youth.

\section{Introduction}

The functional movement screen (FMS) was developed as a comprehensive preparticipation and pre-season assessment. It consists of seven movement tests designed to assess the quality of fundamental movement patterns and presumably identify functional limitations or asymmetries that reflect combinations of muscle strength, flexibility, range of motion, coordination, balance, and proprioception (Cook et al., 2006a, 2006b). Although the FMS is popular among athletic teams and in other settings, data regarding the reproducibility and validity of its measurements have been lacking. However, studies of the interand intra-rater reliability of the FMS indicated that expert and experienced raters demonstrated better reliability than novice raters (Minick et al.,
2010; Shultz et al., 2013). In addition, other studies have indicated that the FMS demonstrated moderate-to-good inter- and intra-rater reliability among novice raters, with acceptable levels of measurement error (Smith et al., 2013; Teyhen et al., 2012).

Recent studies have reported normative data for the FMS in young adults (Schneiders et al., 2011) and distance runners (Agresta et al., 2014). The FMS has also been used to evaluate and reduce injury risk in Marine officer candidates (O'Connor et al.,2011), and used by sports teams to screen for injury risk during the pre-season (Chorba et al., 2010; Kiesel et al., 2007) and to develop standardized off-season intervention programs to prevent injuries (Kiesel et al., 2011). Thus, the FMS is considered to be an appropriate

\footnotetext{
1 - Department of Human Health, Faculty of Human Health, Aichi Toho University, Nagoya, Japan.

2 - Laboratory of Exercise Science, College of Nursing Art and Science, University of Hyogo, Hyogo, Japan.

3 - Department of Health and Sports Sciences, Faculty of Health and Medical Sciences, Kyoto Gakuen University, Kyoto, Japan.

4 - Graduate School of Education and Human Development, Nagoya University, Nagoya, Japan.
} 
screening tool to simultaneously assess multiple domains of function.

Schneiders et al. (2011) suggested that research employing the FMS in different populations and sports was needed due to the lack of FMS data for specific groups such as a younger population of elementary to high school age. Recent studies have examined the relationships between functional movement patterns during physical activity and the body mass index (BMI) in children (Duncan and Stanley, 2012) and between functional movement patterns and overweight/obesity in children (Duncan et al., 2013). However, although the FMS has been used to establish normative values and determine potential injury risk for young adults and athletes, there are little data in elementary school-age children. In addition, in junior sports, children participate in only one particular sport throughout the year from lower grades of primary school, and consequently, early specialization in sports is inevitable (Moesch et al., 2011). Early specialization was shown to increase risks of overuse injuries and burnout (Brenner, 2007), and thus, implementing injury prevention measures is one of the key tasks related to junior sports.

The purpose of this study was to establish fundamental values for the FMS in a population of mini-basketball players, which is a game for boys and girls who are 11 years old or younger at the time of the competition (FIBA, 2005). Secondary purposes were to examine relationships between functional movement patterns and age, peak height velocity (PHV) and BMI, and to compare functional movement patterns between boys and girls and between individuals with and without a history of injury.

\section{Methods}

\section{Participants}

Seventy-one players (47 boys, 24 girls) in five community-based club teams (three boys' teams, two girls' teams) of mini-basketball in Kobe, Japan, participated in this study. Mini-basketball is a game for boys and girls who are 11 years old or younger at the time of the competition (FIBA, 2005). However, in Japanese mini-basketball, elementary school-age players are 12 years old or younger. Participants were elementary school third grade (9 years old) to sixth grade (12 years old) students with a mean \pm standard deviation age of $11.09 \pm 0.8$ years (boys: $11.03 \pm 0.8$ years; girls: $11.21 \pm 0.8$ years), body height of $145.2 \pm 9.7$ cm (boys: $144.0 \pm 10.0 \mathrm{~cm}$; girls: $147.6 \pm 8.9 \mathrm{~cm}$ ), and body mass of $36.0 \pm 7.8 \mathrm{~kg}$ (boys: $35.5 \pm 8.2 \mathrm{~kg}$; girls: $36.9 \pm 6.9 \mathrm{~kg}$ ) (Table 1). They participated in practices and games two to three times a week in their club teams (mean practice frequency: $58.4 \pm$ 12.8 sessions per year, mean game frequency: 44.7 \pm 17.3 games per year). Exclusion criteria included (Paszkewicz et al., 2013; Schneiders et al., 2011): the use of a mobility aid or prophylactic device such as a knee or ankle brace, recent ( $\leq 6$ weeks) musculoskeletal impairment or injury or head injury, or any musculoskeletal surgery within the past year that was likely to affect motor performance on the FMS. Children who were diagnosed with any form of developmental disorder likely to influence motor performance were also not included (i.e., developmental coordination disorder, dyspraxia, dyslexia, Asperger's syndrome, and autism) (Duncan et al., 2013). A consent form was completed for each participant by the parent or guardian. This study was approved by the institutional review board at the Aichi Toho University.

\section{Design and Procedures}

Data collection took place between July and August, 2013, at each club team gymnasium in Kobe, Japan. The research team set up a total of 10 stations: three stations for body height, sitting height, and body mass, and seven stations to conduct the FMS. Participants proceeded through the three anthropometric stations and seven FMS evaluation stations, at which time they were educated on successful performance of the station and then assessed. The data were collected by nine raters from the research team, including two experienced raters (a certified athletic trainer and a certified personal trainer) and seven novice raters (two nursing students and five strength and conditioning students enrolled in the National Strength and Conditioning Association Japan educational program). All raters participated in in-house training sessions, the administration and scoring of the FMS, review of relevant literature, study of related documentation, and working with several members of the research team, which resulted in standardized data collection procedures. To establish that the nine raters who collected the study data scored the participants in a similar manner, the inter-rater reliability of the 
composite score (intraclass correlation model 3,1) was examined. The intraclass correlation for the nine raters was .994, indicating excellent reliability.

Before testing, we sent a questionnaire regarding injury history to the parent or guardian of each participant. They completed the questionnaire and sent it back to us. Previous injury history included acute or chronic injuries of the ankle or knee joints or other acute or chronic injuries of the lower extremities, including surgery within the last 6 months. Five boys and one girl were excluded from the analysis of the relationship between the composite FMS score and history of injury because of an absence of data regarding injury history.

\section{Anthropometry}

Body mass was measured to the nearest half kilogram using weighing scales (Misaki Co., Ltd, Japan), and height and sitting height were measured to the nearest half centimeter using height (Misaki Co., Ltd, Japan) and sitting height (Sanwa Manufacturing Co., Ltd, Japan) scales, respectively. The BMI was determined as $\mathrm{kg} / \mathrm{m}^{2}$.

PHV was utilized as the biological maturity measure according to the methodology from Gastin and Bennett (2014) and Gastin et al. (2013). Age of PHV is the most commonly used indicator of maturity in longitudinal studies of adolescence, and PHV was predicted using the equation of the previous study (Mirwald et al., 2002). This equation is also a reliable, noninvasive and a practical solution for the measure of biological maturity for adolescent athletes (Mirwald et al., 2002). In boys, maturity offset $=-9.236+0.0002708$ $\times$ leg length $\times$ sitting height $-0.001663 \times$ age $\times$ leg length $+0.007216 \times$ age $\times$ sitting height $+0.02292 \times$ weight-by-height ratio. In girls, maturity offset $=-$ $9.376+0.0001882 \times$ leg length $\times$ sitting height + $0.0022 \times$ age $\times$ leg length $+0.005841 \times$ age $\times$ sitting height $-0.002658 \times$ age $\times$ weight $+0.07693 \times$ weight-by-height ratio. Height measurements were in centimeters and weight measurements were in kilograms; the weight-by-height ratio was multiplied by 100 (Malina et al., 2006). For each participant, maturity offset was added to chronological age to provide an estimate of age at PHV.

\section{Functional Movement Assessment}

The FMS is a pre-participation screening tool evaluating the fundamental movement patterns that underpin performance of all movement (Cook et al., 2006a, 2006b). The FMS consists of seven component tests: deep squat, in-line lunge, hurdle step, shoulder mobility, stability push-up, rotational stability, and active straight-leg raise. These tests challenge an individual's ability to perform basic movement patterns that reflect combinations of muscle strength, flexibility, range of motion, coordination, balance, and proprioception (Cook et al., 2006a, 2006b). The FMS was administered by a trained rater using standardized procedures, instructions, and scoring processes (Cook et al., 2006a, 2006b). Each participant was given three trials on each of the seven tests. Each trial was scored on an ordinal scale ranging from zero to three points based on the quality of movement. For each test, the highest score from the three trials was recorded, and the scores of the component tests were summed, resulting in a composite score ranging from zero to 21 points.

\section{Statistical Analyses}

To provide a comprehensive description of the participants and the FMS data, means and standard deviations were computed for each variable for boys and girls separately, and for all participants combined. The Kolmogorov-Smirnov test confirmed that all variables were nonnormally distributed $(p<.05)$; therefore, nonparametric tests were used for all analyses (Duncan et al., 2013). Relationships between the composite FMS score and BMI, age, PHV, and PHV age were analyzed using Spearman's rank order correlation. Differences in scores for each test and the composite FMS score between boys and girls, and between those who had and had not sustained an injury in the previous 6 months, were investigated using Mann-Whitney $U$ tests. $p$ values below .05 were regarded as significant. The statistical procedures were accomplished using Excel Statistics 2015 for Windows (Version 1.03, Social Survey Research Information Co., Ltd.) and the IBM SPSS Statistics for Windows, version 21.0 (Japanese) (IBM Corp, Armonk, NY, USA).

\section{Results}

The mean composite FMS score was 16.5 \pm 2.4 for boys, $16.5 \pm 1.7$ for girls, and $16.5 \pm 2.2$ for all participants combined (Table 1 ). The composite FMS score was significantly positively correlated 
with age $(r=.312, p=.008$, Figure 1$)$ and significantly negatively correlated with the BMI $(r$ $=-.371, p=.002$, Figure 2). However, the FMS score was not significantly correlated with PHV ( $p$ $=.539)$ or with PHV age $(p=.148)$.

There was no significant difference in the composite FMS score between boys and girls ( $p=$ .922, Table 1). The scores for the different FMS tests are presented in Figure 3 for each gender. There was a significant difference between boys and girls for the trunk stability push-up $(p<.001)$ and rotary stability $(p=.033)$ tests. There was no significant difference in the composite FMS score between those who reported a previous injury and those who did not $(p=.423$, Table 2$)$.

Table 1

Physical characteristics (age = years; body height $=\mathrm{cm}$; sitting height $=\mathrm{cm} ;$ body mass $=\mathrm{kg}$; body mass index $(B M I)=k g / m^{2} ;$ peak height velocity $(P H V) ;$ PHV age = years;

functional movement screen (FMS) score) for boys, girls, and combined (boys and girls) group in Japanese mini-basketball players.

\begin{tabular}{|c|c|c|c|}
\hline \multirow[t]{2}{*}{ Variable } & \multicolumn{2}{|c|}{ Gender } & \multirow[t]{2}{*}{ Combined } \\
\hline & Boys & Girls & \\
\hline $\mathrm{N}$ & 47 & 24 & 71 \\
\hline Age (years) & $11.03 \pm 0.8$ & $11.21 \pm 0.8$ & $11.09 \pm 0.8$ \\
\hline Body Height $(\mathrm{cm})$ & $144.0 \pm 10.0$ & $147.6 \pm 8.9$ & $145.2 \pm 9.7$ \\
\hline Sitting Height $(\mathrm{cm})$ & $76.8 \pm 5.0$ & $79.7 \pm 4.5$ & $77.7 \pm 5.0$ \\
\hline Body Mass (kg) & $35.5 \pm 8.2$ & $36.9 \pm 6.9$ & $36.0 \pm 7.8$ \\
\hline BMI $\left(\mathrm{kg} / \mathrm{m}^{2}\right)$ & $17.0 \pm 2.4$ & $16.8 \pm 1.9$ & $16.9 \pm 2.2$ \\
\hline PHV & $-2.38 \pm 0.8$ & $-0.64 \pm 0.9$ & $-1.79 \pm 1.2$ \\
\hline PHV Age (years) & $13.41 \pm 0.5$ & $11.85 \pm 0.5$ & $12.88 \pm 0.9$ \\
\hline FMS Score & $16.5 \pm 2.4$ & $16.5 \pm 1.7$ & $16.5 \pm 2.2$ \\
\hline
\end{tabular}

\section{Table 2}

The composite functional movement screen (FMS) scores between previous injury history and no injury history for boys, girls, and combined (boys and girls) group in Japanese mini-basketball players.

\begin{tabular}{|c|c|c|c|c|}
\hline \multirow[t]{3}{*}{ Variable } & \multicolumn{4}{|c|}{ FMS Scores } \\
\hline & \multicolumn{2}{|c|}{ Previous Injury History } & \multicolumn{2}{|c|}{ No Injury History } \\
\hline & $\mathrm{N}$ & Mean \pm SD & $\mathrm{N}$ & Mean \pm SD \\
\hline Boys & 9 & $1016.1 \pm 2.2$ & 33 & $1616.8 \pm 2.4$ \\
\hline Girls & 7 & $16.4 \pm 2.6$ & 16 & $16.6 \pm 1.3$ \\
\hline Combined & 16 & $16.3 \pm 2.3$ & 49 & $16.8 \pm 2.1$ \\
\hline
\end{tabular}




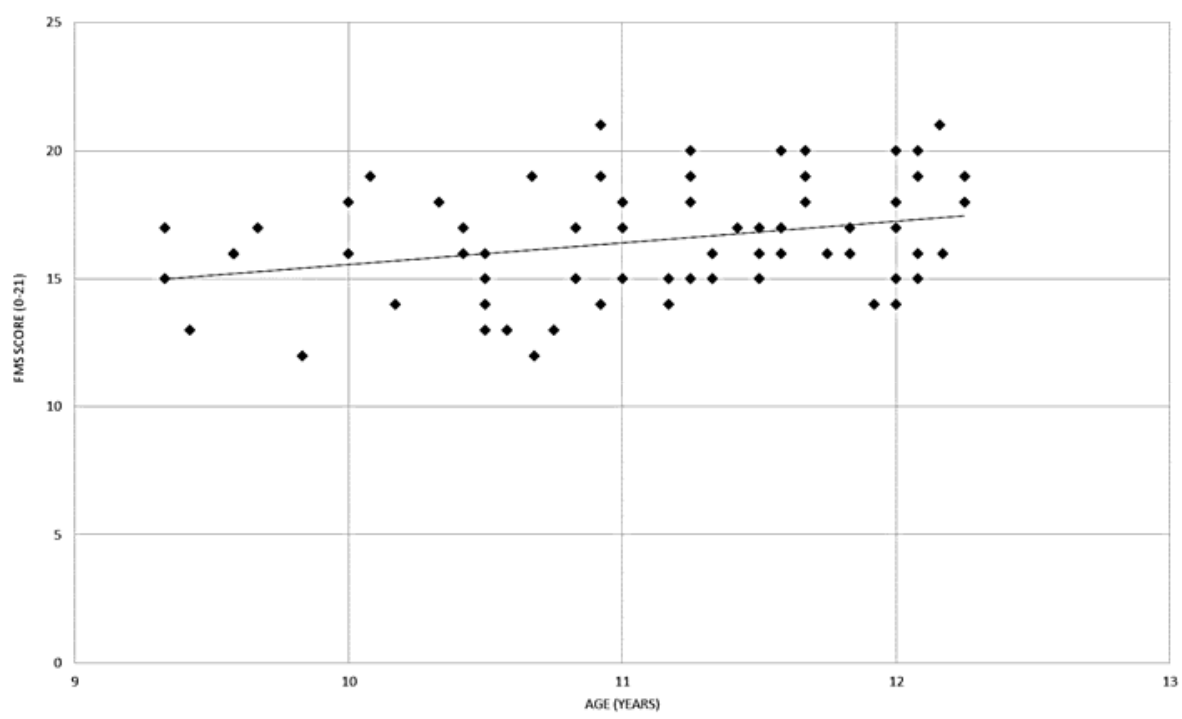

Figure 1

Relationship between the composite functional movement screen

(FMS) score and age in Japanese mini-basketball players.

The composite FMS score was significantly positively correlated with age $(r=.312, p=.008)$.

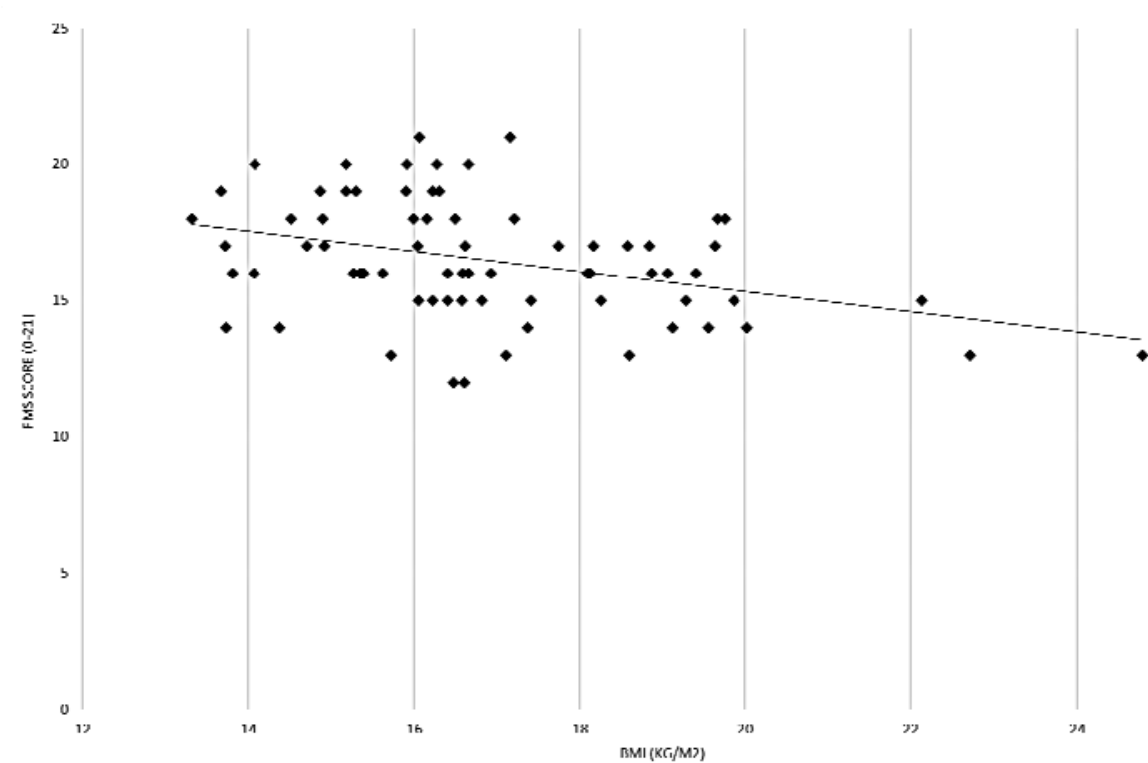

Figure 2

Relationship between the composite functional movement screen (FMS) score and body mass index (BMI) in Japanese mini-basketball players.

The composite FMS score was significantly negatively correlated with the BMI ( $r=-.371, p=.002)$. 


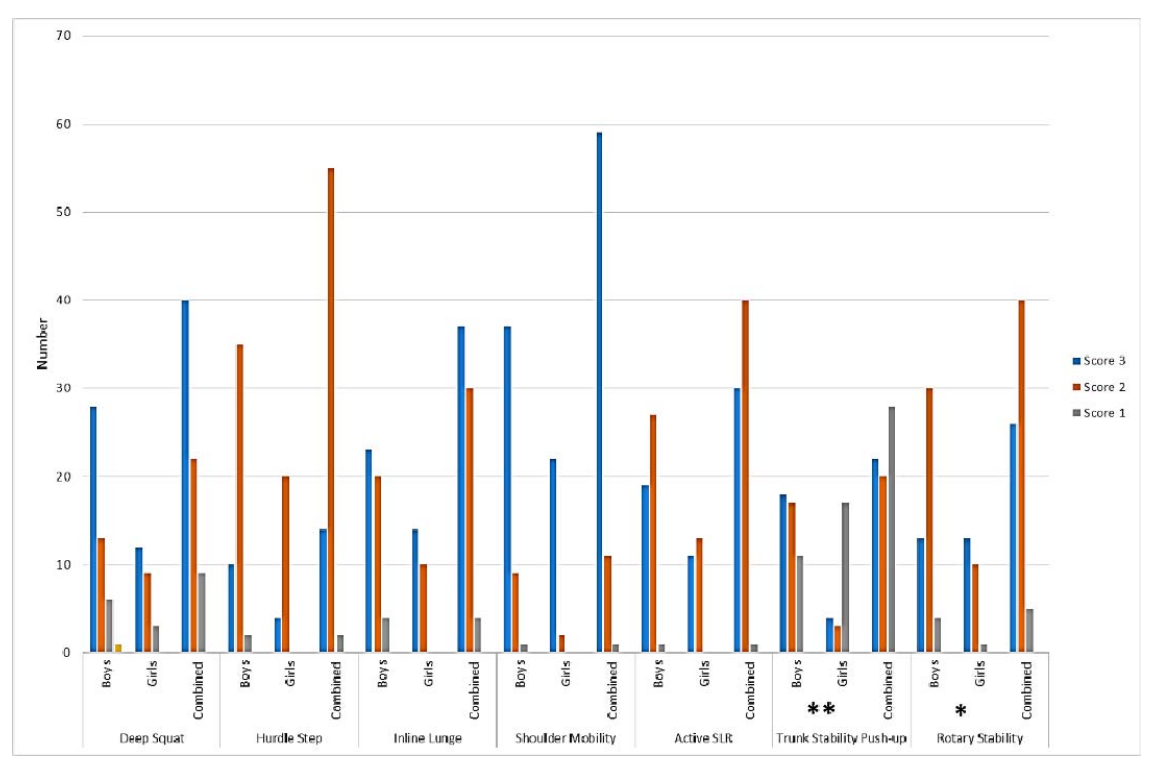

\section{Figure 3}

Score distributions for each individual functional movement screen (FMS)

test (deep squat, hurdle step, inline lunge, shoulder mobility, active straight leg raise (SLR), trunk stability push-up, rotary stability) among boys, girls, and combined (boys and girls) group in Japanese mini-basketball players.

** Significant difference between boys and girls for the trunk stability push-up test $(p<.001)$.

*Significant difference between boys and girls for the rotary stability test $(p=.033)$.

\section{Discussion}

This study provides normative data for the FMS in elementary school-age mini-basketball players. In addition, we identified associations between the composite FMS score and age, PHV, and BMI and compared the FMS score between boys and girls, and between children in the same age group with and without a history of injury.

The mean composite score (16.5) in this study was slightly higher than that previously reported in children with normal body mass (14.7 and 15.5) (Duncan and Stanley, 2012; Duncan et al., 2013). It might be expected that minibasketball players score better owing to habitual physical activity such as practice and games in mini-basketball two to three times a week over the course of each year. The higher composite score seen in this study supports previous research by
Duncan and Stanley (2012), which identified a positive relationship between the composite FMS score and habitual physical activity.

This study demonstrated that composite FMS score was significantly and positively related to age in mini-basketball players. Paszkewicz et al. (2013) indicated that post-pubescent participants had a significantly higher composite FMS score than prepubescent participants, and demonstrated differences in the FMS score across the maturation range. Their study suggested that after the pubertal growth spurt, there was an increase in muscular strength, flexibility, proprioception, and coordination in both genders (Paszkewicz et al., 2013). Increases in these factors allow post-pubescent adolescents to perform more complex movement patterns used in common daily activities and sports (Minick et al., 
2010). In addition, using this capability, postpubescent adolescents may possess better muscle activation patterns during movements, and therefore have better dynamic control of their bodies than prepubescent and early-pubescent adolescents (Paszkewicz et al., 2013). Thus, the results of our study suggest that fundamental movement patterns may be influenced by age and maturation in addition to intrinsic factors such as functional capacity, muscle activation, neuromuscular control, and mobility.

On the other hand, we demonstrated that the composite FMS score was not significantly related to PHV or PHV age in mini-basketball players. In general, PHV typically occurs at around the age of 12 years in females and 14 years in males (Lloyd et al., 2014), which is consistent with our results in mini-basketball players (Table 1). At this age, children of the same chronological age can differ markedly with respect to biological maturity, and there is significant inter-individual variance in the level (magnitude of change), timing (onset of change), and tempo (rate of change) of biological maturation (Lloyd et al., 2014; Malina et al., 2005). Thus, the lack of correlation between the composite FMS score and PHV in our results may be influenced by individual differences in biological maturation.

We demonstrated that the composite FMS score was negatively related to the BMI, with functional movement being poorer in minibasketball players with a higher BMI. In this respect, the results of this study confirm previous reports that the composite FMS score is significantly and inversely related to the BMI in 10 to 11-year-old children (Duncan and Stanley, 2012) and 7 to 10-year-old children (Duncan et al., 2013). It was suggested that excess weight resulted in functional limitations and indicated that children displayed poor functional movement as a consequence of excess weight (Duncan and Stanley, 2012). Therefore, the data in this study extend current knowledge on poor functional movement performance with a higher BMI in mini-basketball players as well as in children in the general population.

In this study, there was no significant difference in the composite FMS score between boys and girls, yet there were significant gender differences on two individual FMS tests. In previous studies of young active adults (age range
18-40 years, mean age $21.9 \pm 3.7$ years) (Schneiders et al., 2011) and healthy distance runners (age range 22-54 years, mean age $34.8 \pm$ 7.7 years) (Agresta et al., 2014), there was no gender difference in the composite FMS score. In other studies (Duncan and Stanley, 2012; Duncan et al., 2013), there was no gender difference in the composite FMS score of 10 to 11-year-old children with different weight status (normal, overweight, and obese). Paszkewicz et al. (2013) performed functional and static evaluation measures (FMS and the Beighton and Horan joint mobility index, respectively) in adolescent athletes (age range 814 years, mean age $11.5 \pm 2.0$ years) and found no significant gender difference in the composite FMS score or Beighton and Horan joint mobility index score. These previous studies are consistent with our study, and suggest that there is no gender difference in the composite FMS score in junior players aged 14 years old or less or in young adults aged 18 years old or more. Based on these results, Schneiders et al. (2011) suggested that the FMS could be used to compare individuals in mixed populations.

On the other hand, Anderson et al. (2015) quantified the FMS score in healthy male and female secondary school athletes (age range 8-14 years) and found that the composite FMS score was significantly lower in females than in males, which is inconsistent with our findings. The FMS score for post-pubescent male athletes might be increasing at this age, because PHV typically occurs around the age of 14 years in males (Lloyd et al., 2014). Thus, further studies are necessary of FMS scores in individuals aged 14 to 18 years old.

It is important to note that although there was no difference in this study in the composite FMS score of boys and girls, significant differences were apparent between boys and girls on two individual FMS tests. Boys were better than girls on the trunk stability push-up test and the rotary stability test. The trunk stability pushup test is associated with upper-body strength and stability, including core stability in the sagittal plane, and the rotary stability test is associated with transverse plane (rotational) core stability (Cook et al., 2006b). Thus, the results of this study suggest that boys aged 9 to 12 years old have a stronger upper-body and better trunk muscular control than girls of the same age. Similarly, Schneiders et al. (2011) demonstrated 
that males were significantly stronger than females on the trunk stability push-up and rotary stability tests in a study that investigated 209 physically active individuals, supporting the findings of our study. In another study of healthy distance runners by Agresta et al. (2014), there was no significant difference in the composite FMS score across genders, but males scored significantly higher than females on the deep squat and trunk stability push-up tests. Based on these results, we suggest that individual FMS tests can be used to compare differences in specific functional movements across genders in different populations.

This study demonstrated that there was no significant difference in the composite FMS score between participants who reported a previous injury and those who did not, which is consistent with previous studies (Agresta et al., 2014; Schneiders et al., 2011). They indicated that runners with a history of injury had higher mean composite scores than those without history of injury, yet this difference was not significant (Agresta et al., 2014). The higher scores in previously injured runners may be a result of focused treatment, such as physical therapy or strength training, received for a particular injury (Agresta et al., 2014). On the other hand, Kiesel et al. (2007) retrospectively examined the relationship between the FMS score and the likelihood of serious injury (being an injured reserve for at least 3 weeks) in professional American football players and reported that players with a composite FMS score of 14 or less (out of a maximum of 21) were more likely to suffer a serious injury. Additionally, Chorba et al. (2010) examined whether compensatory movement patterns predisposed female collegiate athletes (soccer, volleyball, and basketball) to injury, and found that a score of 14 or less on the FMS increased the risk of injury. After excluding athletes with a history of anterior cruciate ligament reconstruction, linear regression analysis revealed a significant negative correlation between the FMS score and injury, and an FMS score of 14 or less resulted in a four-fold increase in the risk of lower-extremity injury (Chorba et al., 2010). However, our study showed no significant difference in the FMS score between participants with a previous injury and those without. History of injury included acute injury of the lower extremity, such as ankle or knee injury, in addition to chronic injury such as OsgoodSchlatter disease, which frequently occurs during growth and development. There were no serious injuries reported in the elementary school-age mini-basketball players. This may explain the lack of significant difference in the FMS score between participants with a previous injury and those without.

The FMS is designed to measure movement capacity and requires adequate muscle strength and activation, neuromuscular control, and core stability for successful completion of the movement tasks. The FMS has been growing in popularity as a pre-participation evaluation. However, the FMS has never been reported in elementary school-age mini-basketball players. Mini-basketball has the second largest number of elementary school-age participants (approximately 150,000 players per year), following junior soccer (approximately 320,000 players per year) and is one of very popular junior sports in Japan (Japan Basketball Association, 2016; Kuzuhara et al., 2016). Prevention of injury during adolescence should be a major priority of coaches, athletic trainers (or physiotherapists), strength and conditioning coaches, and players. Individual differences and drastic musculoskeletal alterations in biological maturation during pubertal growth mean that it is necessary for coaches to understand individual variance in elementary school-age players. Using the FMS to detect musculoskeletal alterations in children of elementary school age would allow clinicians to identify elementary school-age players who would benefit from individualized neuromuscular training programs to prevent future injuries. Moreover, for those coaching elementary school-age players in mini-basketball, the FMS is a very useful tool to provide each player with appropriate individual physical conditioning.

A limitation of our study is its limited power to determine whether truly significant differences exist between different groups such as different sports teams, experience levels, or injury histories. Future studies should incorporate longitudinal, prospective research investigating FMS scores across pubertal groups in a larger elementary school-age population. Future research should also prospectively evaluate the 
injury rate in relation to FMS scores in an elementary school-age population.

This is the first study to evaluate FMS scores in elementary school-age mini-basketball players. This study determined normative values for children of this age in mini-basketball. Age and the BMI were significantly associated with better and poorer functional movement, respectively. However, the composite FMS score was not significantly related to PHV or PHV age in mini-basketball players. Although there was no difference in this study in the composite FMS score of boys and girls, significant differences were apparent between boys and girls on two individual FMS tests. Boys had higher scores on the trunk stability push-up test and the rotary stability test than girls. In addition, there was no significant difference in the composite FMS score between participants who reported a previous injury and those who did not.

\section{Acknowledgements}

This research was supported by JSPS KAKENHI (C) Grant Number JP25350738.

\section{References}

Agresta C, Slobodinsky M, Tucker C. Functional Movement Screen ${ }^{\mathrm{TM}}$-Normative values in healthy distance runners. Int J Sports Med, 2014; 35: 1203-1207

Anderson BE, Neumann ML, Huxel Bliven KC. Functional movement screen differences between male and female secondary school athletes. J Strength Cond Res, 2015; 29: 1098-1106

Brenner JS. Overuse injuries, overtraining, and burnout in child and adolescent athletes. Pediatrics, 2007; 119: 1242-1245

Chorba RS, Chorba DJ, Bouillon LE, Overmyer CA, Landis JA. Use of a functional movement screening tool to determine injury risk in female collegiate athletes. North Am J Sports Phys Ther, 2010; 5: 47-54

Cook G, Burton L, Hoogenboom B. Pre-participation screening: The use of fundamental movements as an assessment of function - Part 1. North Am J Sports Phys Ther, 2006a; 1: 62-72

Cook G, Burton L, Hoogenboom B. Pre-participation screening: The use of fundamental movements as an assessment of function - Part 2. North Am J Sports Phys Ther, 2006b; 1: 132-139

Duncan MJ, Stanley M. Functional movement is negatively associated with weight status and positively associated with physical activity in British primary school children. J Obesity, 2012; 697563, doi: $10.1155 / 2012 / 697563$

Duncan MJ, Stanley M, Wright SL. The association between functional movement and overweight and obesity in British primary school children. BMC Sports Sci Med Rehabil, 2013; 5: 11

FIBA. Mini basketball rules 2005.

Available at: http://www.fiba.com/downloads/Rules/2005_mini_bask_rule.pdf

Gastin PB, Bennett G. Late maturers at a performance disadvantage to their more mature peers in junior Australian football. J Sports Sci, 2014; 32: 563-571

Gastin PB, Bennett G, Cook J. Biological maturity influences running performance in junior Australian football. J Sci Med Sport, 2013; 16: 140-145

Japan Basketball Association. Enrollment of athletes in 2016.

Available at : http://www.japanbasketball.jp/wp-content/uploads/athlete_2016.pdf ; accessed on 30.03.2017.

Kiesel K, Plisky P, Butler R. Functional movement test scores improve following a standardized off-season intervention program in professional football players. Scand J Med Sci Sports, 2011; 21: 287-292

Kiesel K, Plisky PJ, Voight ML. Can serious injury in professional football be predicted by a preseason functional movement screen? North Am J Sports Phys Ther, 2007; 2: 147-158

Kuzuhara K, Shibata M, Uchida R. Injuries in Japanese mini-basketball players during practices and games. J Athl Train, 2016; 51: 1022-1027 
Lloyd RS, Oliver JL, Faigenbaum AD, Myer GD, De Ste Croix MBA. Chronological age vs. biological maturation: Implications for exercise programming in youth. J Strength Cond Res, 2014; 28: 1454-1464

Malina RM, Claessens AL, Van Aken K, Thomis M, Lefevre J, Philippaerts R, Beunen GP. Maturity offset in gymnasts: application of a prediction equation. Med Sci Sports Exerc, 2006; 38: 1342-1347

Malina RM, Cumming SP, Morano PJ, Barron M, Miller SJ. Maturity status of youth football players: A noninvasive estimate. Med Sci Sports Exerc, 2005; 37: 1044-1052

Minick KI, Kiesel KB, Burton L, Taylor A, Plisky P, Butler RJ. Interrater reliability of the functional movement screen. J Strength Cond Res, 2010; 24: 479-486

Mirwald RL, Baxter-Jones ADG, Bailey DA, Beunen GP. An assessment of maturity from anthropometric measurements. Med Sci Sports Exerc, 2002; 34: 689-694

Moesch K, Elbe AM, Haugu MLT, Wikman JM. Late specialization: the key to success in centimeters, grams, or seconds (cgs) sports. Scand J Med Sci Sports, 2011; 21: e282-e290

O'Connor FG, Deuster PA, Davis J, Pappas CG, Knapik JJ. Functional movement screening: Predicting injuries in officer candidates. Med Sci Sports Exerc, 2011; 43: 2224-2230

Paszkewicz JR, McCarty CW, Van Lunen BL. Comparison of functional and static evaluation tools among adolescent athletes. J Strength Cond Res, 2013; 27: 2842-2850

Schneiders AG, Davidsson A, Horman E, Sullivan SJ. Functional movement screen normative values in a young, active population. Int J Sports Phys Ther, 2011; 6: 75-82

Shultz R, Anderson SC, Matheson GO, Marcello B, Besier T. Test-retest and interrater reliability of the functional movement screen. J Athl Train, 2013; 48: 331-336

Smith CA, Chimera NJ, Wright NJ, Warren M. Interrater and intrarater reliability of the functional movement screen. J Strength Cond Res, 2013; 27: 982-987

Teyhen DS, Shaffer SW, Lorenson CL, Halfpap, JP, Donofry, DF, Walker, MJ, Dugan, JL, Childs JD. The functional movement screen: A reliability study. J Orthop Sports Phys Ther, 2012; 42: 530-540

\section{Corresponding author:}

\section{Kenji Kuzuhara}

Aichi Toho University

Department of Human Health, Faculty of Human Health

3-11 Heiwagaoka, Meito-ku, Nagoya 465-8515, Japan

Phone (international): +81-52-782-1241

Fax (international): +81-52-782-1943

E-mail: kuzuhara.kenji@aichi-toho.ac.jp 